OPEN ACCESS

Edited by: Lei Ye,

National Heart Centre Singapore,

Singapore

Reviewed by:

Zongjin $\mathrm{Li}$,

Nankai University, China Laura lop,

University of Padua, Italy

${ }^{*}$ Correspondence: Yucai Hong

zrhyc@hotmail.com

Xiaoming Zhang

zxm@zju.edu.cn

tThese authors have contributed equally to this work

Specialty section:

This article was submitted to

Stem Cell Research,

a section of the journal

Frontiers in Cell and Developmental

Biology

Received: 20 December 2020

Accepted: 28 February 2021

Published: 22 March 2021

Citation:

Meng K, Cai H, Cai S, Hong Y and Zhang X (2021) Adiponectin Modified BMSCs Alleviate Heart Fibrosis via Inhibition TGF-beta1/Smad in Diabetic Rats. Front. Cell Dev. Biol. 9:644160. doi: 10.3389/fcell.2021.644160

\section{Adiponectin Modified BMSCs Alleviate Heart Fibrosis via Inhibition TGF-beta1/Smad in Diabetic Rats}

\author{
Ke Meng ${ }^{1,2+}$, Huabo Cairt, Simin Cai 1,2, Yucai Hong ${ }^{2 *}$ and Xiaoming Zhang ${ }^{1,2 *}$ \\ 'Department of Anatomy, Sir Run Run Shaw Hospital, School of Medicine, Zhejiang University, Hangzhou, China, \\ ${ }^{2}$ Department of Emergency Medicine, Sir Run Run Shaw Hospital, School of Medicine, Zhejiang University, Hangzhou, China
}

Background: Accumulating evidence suggested that bone marrow mesenchymal stem cells (BMSCs) have therapeutic potential for diabetes and heart diseases. However, the effects of BMSC on reducing myocardial fibrosis need to be optimized. This study aimed to investigate the mechanism of adiponectin (APN) modified BMSCs on myocardial fibrosis in diabetic model in vivo and in vitro.

Methods: The high-fat diet combined with streptozotocin (STZ) injection were used to induced diabetic rat model. H9c2 cells were cultured under a high glucose medium as in vitro model. The BMSCs were modified by APN plasmid or APN small interfering RNA (siRNA), then transplanted to the diabetic rats by a single tail-vein injection, or co-cultured with $\mathrm{H} 9 \mathrm{c} 2$ cells.

Results: We demonstrated that diabetic rats showed typical diabetic symptoms, such as decreased cardiac function, accumulation of pathological lesions and collagen expression. However, these impairments were significantly prevented by the APN modified BMSCs treatment while no effects on APN siRNA modified BMSCs treated diabetic rats. Moreover, we confirmed that APN modified BMSCs could attenuate the expression of TGF-beta1/smad to suppress the myocardial fibrosis in the diabetic rats and high glucose induced $\mathrm{H} 9 \mathrm{c} 2$ cells.

Conclusion: The present results for the first time showed that APN modified BMSCs exerted protection on cardiac fibrosis via inhibiting TGF-beta1/smad signal pathway in diabetic rats. Our findings suggested that APN modified BMSCs might be a novel and optimal therapy for the diabetic cardiomyopathy in future.

Keywords: diabetic cardiomyopathy, adiponectin, bone marrow mesenchymal stem cells, TGF-beta1, myocardial fibrosis

Abbreviations: APN, adiponectin; BMSCs, bone marrow mesenchymal stem cells; DCM, diabetic cardiomyopathy; DMEM, Dulbecco's modified Eagle's medium; FS, fractional shortening; GFP, green fluorescent protein; HE, hematoxylin and eosin; LV, lentivirus vector; LVIDD, left ventricular internal diameter end diastole; LVIDs, left ventricular internal diameter end systole; LVEF, left ventricular ejection fraction; MOI, multiplicity of infection; SD, Sprague-Dawley; SDS-PAGE, sodium dodecyl sulfate-polyacrylamide gel electrophoresis; siRNA, small interfering RNA; STZ, streptozotocin; TGF- $\beta$, transforming growth factor-beta. 


\section{INTRODUCTION}

Diabetes mellitus and its complications become a dominant public health problem (American Diabetes Association, 2012). Clinical cases suggested that heart failure incidence was significantly higher in patients with diabetes (Cavender et al., 2015). The studies revealed that hyperglycemia could directly injure cardiomyocytes leading to diabetic cardiomyopathy (DCM) (Seferovic and Paulus, 2015; Jia et al., 2018b). DCM is one of diabetic complications that causes heart failure in patients with diabetes (Wang et al., 2018). The pathogenesis of DCM is complex, and cardiomyocyte fibrosis is the main distinct pathological features of DCM (Chang et al., 2016; Faramoushi et al., 2016). Biopsies from diabetic patients with heart failure indicated that cardiac fibrosis might play a vital role in the DCM (Falcao-Pires et al., 2011). Cardiac fibrosis contributes to cardiac remodeling and finally leads to the progression of heart failure (Jia et al., 2018a). The transforming growth factor-beta (TGF- $\beta$ ) superfamily serves multiple roles in cell differentiation, proliferation and fibrosis. The TGF- $\beta$ s have three isoforms $(\beta 1, \beta 2$, and $\beta 3)$ in mammals (Travis and Sheppard, 2014), and TGF- $\beta 1$ is implicated in fibrosis (Oruqaj et al., 2015). TGF- $\beta 1$ over-expressed in myocardium induced stable hypertrophy to heart failure. Upregulation of TGF- $\beta 1$ was related to fibrosis in diabetic heart (Koitabashi et al., 2011). High glucose could increase TGF- $\beta 1$ activity and downstream canonical Smad signaling that increased interstitial fibrosis and cellular hypertrophy in the heart of diabetic rats (Bugyei-Twum et al., 2014). Moreover, TGF$\beta 1 /$ Smad may play an important role in fibrogenesis of DCM (Shen et al., 2014).

Bone marrow mesenchymal stem cells (BMSCs) are multipotent stem cells and emerged protection through paracrine related factors and immunomodulatory to host cells (Jing et al., 2014). The scientists found that BMSCs could improve cardiac functions against DCM (Li et al., 2008). However, the effects of BMSCs on reducing cardiac fibrosis still need to be optimized. Interestingly, the adiponectin (APN) that secreted by adipocytes had anti-insulin resistance, antiatherosclerotic, and anti-inflammatory effects (Wang et al., 2013). Furthermore, APN-knockout mice could enhance carbon tetrachloride-induced liver fibrosis (Nishihara et al., 2006) and renal fibrosis (Tian et al., 2018), while increasing APN expression attenuated liver and renal fibrosis. Therefore, we hypothesized a novel therapeutic effect of the APN modified BMSCs on DCM. The present study aimed to investigate the effects and possible signal pathways involved in APN modified BMSCs on cardiac structural and functional improvement of diabetic models in vivo and in vitro.

\section{MATERIALS AND METHODS}

\section{Ethics Statement}

The animal experiments were performed in accordance with the guidelines of the Institutional Animal Care and Use and approved by the Committee of Animal Experiment Center of Zhejiang
University (Hangzhou, China). The best efforts have been made to minimize the number and the suffering of the rats.

\section{Primary BMSCs Culture and Characterization}

As our previous study reported (Wang et al., 2016), the primary BMSCs were harvested from the femurs of 2-weekold Sprague-Dawley (SD) female rats that purchased from Shanghai laboratory animal center (Shanghai, China). The rats were anesthetized with sodium pentobarbital $(40 \mathrm{mg} / \mathrm{kg})$. The bone marrow cavity was flushed and then cultured in Dulbecco's modified Eagle's medium (DMEM, Catalog: 11885-084, Gibco, Invitrogen, United States) with 10\% (V/V) fetal bovine serum (Catalog: 10099-141, Gibco, Invitrogen, United States) and a mixture of $1 \%$ penicillin and streptomycin (Catalog: 10378016, Invitrogen, United States) and maintained in a tri-gas incubator (Thermo Fisher Scientific, Marietta, $\mathrm{OH}$, United States) composed of $5 \% \mathrm{CO}_{2}$ and $95 \%$ air at $37^{\circ} \mathrm{C}$ and changed the fresh medium every 2 days.

\section{Lentivirus Vector Construction and Infection of BMSCs}

Recombinant lentivirus vector (LV) containing green fluorescent protein (GFP) as LV5-APN-GFP and LV3-APN-small interfering RNA (siRNA)-GFP, they were designed and constructed by Shanghai GenePharma Technology Co., Ltd. (Shanghai, China), and LV3-vehicle-GFP virus that expresses GFP only was also constructed as a control (Sanber et al., 2015). The target gene APN (Gene Bank accession NM_031012.1) fragment was inserted into the NotI/BamHI site on the LV5 vector. The target siRNA against rat APN gene was designed as following: $5^{\prime}$-GGATCTCGTTGTTGGGCTTTA-3'. The lentivirus was determined to be $1 \times 10^{8} \mathrm{PFU} / \mathrm{ml}$ by using a titration kit, and the lentivirus vector was stored at $-80^{\circ} \mathrm{C}$ until use. BMSCs $\left(5 \times 10^{5}\right.$ cells) were seeded into the 6-well plate, incubated overnight, and then transfected with LV3-GFP, LV5-APN-GFP, and LV3-APNsiRNA-GFP for $72 \mathrm{~h}$ as BMSCs/Vehicle, BMSCs/APN+, and BMSCs/APN - separately (Sanber et al., 2015). The multiplicity of infection (MOI) was 100. Transduction efficiency of APN was examined after $48 \mathrm{~h}$ by fluorescence microscope (BX-53, Olympus Corp., Tokyo, Japan). The expression of APN was detected by western blot analysis.

\section{Animal Models Induction}

Male SD rats were obtained from Shanghai laboratory animal center (Shanghai, China). The rats were divided into six groups at random: the control group, the DM (diabetic rat) group, the BMSCs/Vehicle group, the BMSCs/APN+ group, and the BMSCs/APN- group $(n=10)$. After $18 \mathrm{~h}$ of fast, the rats were given a single intraperitoneal injection of freshly prepared streptozotocin (STZ; $65 \mathrm{mg} / \mathrm{kg}$ body weight) (Catalog: S8050, Solarbio, Beijing, China) dissolved in 0.01 M citrate buffer ( $\mathrm{pH}$ 4.5) for diabetes modeling, while the control rats were intraperitoneally injected with equal amounts of citrate buffer ( $\mathrm{pH}$ 4.5). A total of $72 \mathrm{~h}$ after injection, the fasting blood glucose was determined using an analyzer 
(Accu-Chek ${ }^{\circledR}$, Roche, Mannheim, Germany). Rats with blood glucose levels above $16.7 \mathrm{mmol} / \mathrm{L}$ were considered successful diabetic models for further study (Hasegawa et al., 2010). One week after STZ injection, the $200 \mu \mathrm{l}$ PBS including $4 \times 10^{6}$ BMSCs, BMSCs/Vehicle, BMSCs/APN+, and BMSCs/APNwere injected into the diabetic rat by a single tail-vein injection as the BMSCs group, the BMSCs/Vehicle group, the BMSCs/APN+ group, and the BMSCs/APN- group, respectively.

\section{Echocardiography Assay for the Cardiac Function and Metabolic Indexes Analysis of All Groups Rats}

Six weeks after transplantation, the cardiac function was estimated through a M9 echocardiography machine (Mindray, Shenzhen, China). We anesthetized all rats with an intraperitoneal sodium pentobarbital $(40 \mathrm{mg} / \mathrm{kg})$ and removed the chest hair by a pet razor. A $30-\mathrm{MHz}$ probe was then used to carry out the exam (Scherrer-Crosbie et al., 1998). The left ventricular internal diameter end diastole (LVIDD), left ventricular internal diameter end systole (LVIDs), left ventricular ejection fraction (LVEF), and fractional shortening (FS) were measured by an echocardiography doctor who blinded to all groups. After echocardiography assay, the body weight and fasting blood glucose were recorded on the basis of fasting for $8 \mathrm{~h}$. All rats were anesthetized with an intraperitoneal sodium pentobarbital $(40 \mathrm{mg} / \mathrm{kg})$. We removed all rat's hearts, and recorded heart weight, then for subsequent analysis.

\section{Masson, Hematoxylin and Eosin and Immunohistochemistry Staining}

For pathological analysis, the heart sections were respectively subjected to Masson and hematoxylin and eosin (HE) staining (Wang et al., 2014). The hearts of five animals from each group were removed and fixed in $4 \%$ paraformaldehyde, then embedded in paraffin and sliced into sections ( $8 \mu \mathrm{m}$ thick). We used $\mathrm{HE}$ (Catalog: G1005, Solarbio, Beijing, China), Masson's trichrome staining (Catalog: G1006, Servicebio, Wuhan, China), and immunohistochemistry staining to determine the left ventricular wall thickness, the collagen area, and the cross-sectional area of the cardiomyocytes. All images of the sections were collected by a light microscope (BX-53, Olympus Corp., Tokyo, Japan), and analyzed with Image-Pro Plus 6.0 software (Media Cybernetics, MD, United States) for positive area measurement of whole vision field in each figure under $400 \times$ microscope to analyze the positive area of Masson's trichrome staining that relative to whole myocardium area (\%).

Expression and localization of the target protein were observed by immunohistochemistry methods. The serial heart sections were incubated with primary antibodies overnight at $4^{\circ} \mathrm{C}$, including TGF- $\beta 1$ (Catalog: GB11179, 1:100, Boster Bio, Wuhan, China); Smad2 (Catalog: BA4557, 1:100, Boster Bio, Wuhan, China); Smad3 (Catalog: BA4559, 1:100, Boster Bio, Wuhan, China); Collagen I (Catalog: BA0325, 1:100, Boster Bio, Wuhan, China), and Collagen III (Catalog: BA0326, 1:100, Boster Bio, Wuhan, China). Sections were washing with PBS and subsequently incubated with biotinylated and affinitypurified IgG secondary antibodies at room temperature for $4 \mathrm{~h}$. Images were visualized by a light microscope (BX-53, Olympus Corp., Tokyo, Japan) and analyzed by Image-Pro Plus 6.0 (Media Cybernetics, Maryland, United States) for positive area measurement of whole vision field in each figure under $400 \times$ microscope to analyze the positive area of collagen I and collagen III Immunostaining that relative to whole myocardium area $(\%)$.

\section{H9c2 Co-cultured With Modified BMSCs Under High Glucose Medium}

Rat cardiomyocyte cell line H9c2 cells were cultured in DMEM medium (Catalog: 11885-084, Gibco, Invitrogen, United States) with $10 \%(\mathrm{~V} / \mathrm{V}$ ) fetal bovine serum (Catalog: 10099-141, Gibco, Invitrogen, United States), and supplement with $25 \mathrm{mM}$ glucose (control group) or with $50 \mathrm{mM}$ high glucose (DM group), at a humidified atmosphere containing 5\% $\mathrm{CO}_{2}$ and $95 \%$ air. The above BMSCs $\left(5 \times 10^{5} /\right.$ well $)$ were seeded in the insert chamber of 6-well $0.4 \mu \mathrm{m}$ transwell system, then co-cultured with $5 \times 10^{5} /$ well H9c2 in DMEM medium with $50 \mathrm{mM}$ high glucose as DM+ BMSCs group, DM+ BMSCs/Vehicle group, $\mathrm{DM}+\mathrm{BMSCs} / \mathrm{APN}+$ group, and $\mathrm{DM}+\mathrm{BMSCs} / \mathrm{APN}-$ group, respectively. After $7 \mathrm{~d}, \mathrm{H} 9 \mathrm{c} 2$ cells were washed twice with PBS, lysed in Western and IP buffer (Catalog: P0013, Beyotime Institute of Biotechnology, Shanghai, China), then stored at $-80^{\circ} \mathrm{C}$ for western blot analysis.

\section{Western Blot Analysis}

Proteins expression of rat's hearts and co-cultured cells were analyzed by western blot. The BCA protein concentration assay kit was used for protein quantification. Equal amounts of protein were separated using $10 \%$ sodium dodecyl sulfatepolyacrylamide gel electrophoresis (SDS-PAGE) and transferred from the gel to nitrocellulose membrane, blocked for $1 \mathrm{~h}$ with $5 \%$ skimmed milk in Tris-buffered saline containing $0.1 \%$ Tween-20 at room temperature. The membranes were incubated overnight at $4^{\circ} \mathrm{C}$ with the primary antibodies TGF- $\beta 1$ antibody (Catalog: 3711, 1:1000); Smad2 (Catalog: 5339, 1:1000); phosphoSmad2 (Catalog: 3108, 1:1000); Smad3 (Catalog: 9523, 1:1000), and phospho-Smad3 (Catalog: 9520, 1:1000) purchased from Cell Signaling Technology (Danvers, MA, United States). Actin (Catalog: A2228, 1:5000) was purchased from Sigma-Aldrich (Merck, KGaA, Darmstadt, Germany). After incubation for 16 h, the membranes were washed with TBST and incubated with infrared labeled secondary antibody for $1 \mathrm{~h}$ at room temperature and washed three times with TBST. Immunoblotted bands were analyzed by CLx Odyssey infrared imaging system (Li-COR biosciences, United States).

\section{Statistical Analysis}

Values were expressed as mean \pm standard deviation (SD). All parameters were statistically performed by one-way analysis of variance (ANOVA).p $<0.05$ was considered statistically significant. 


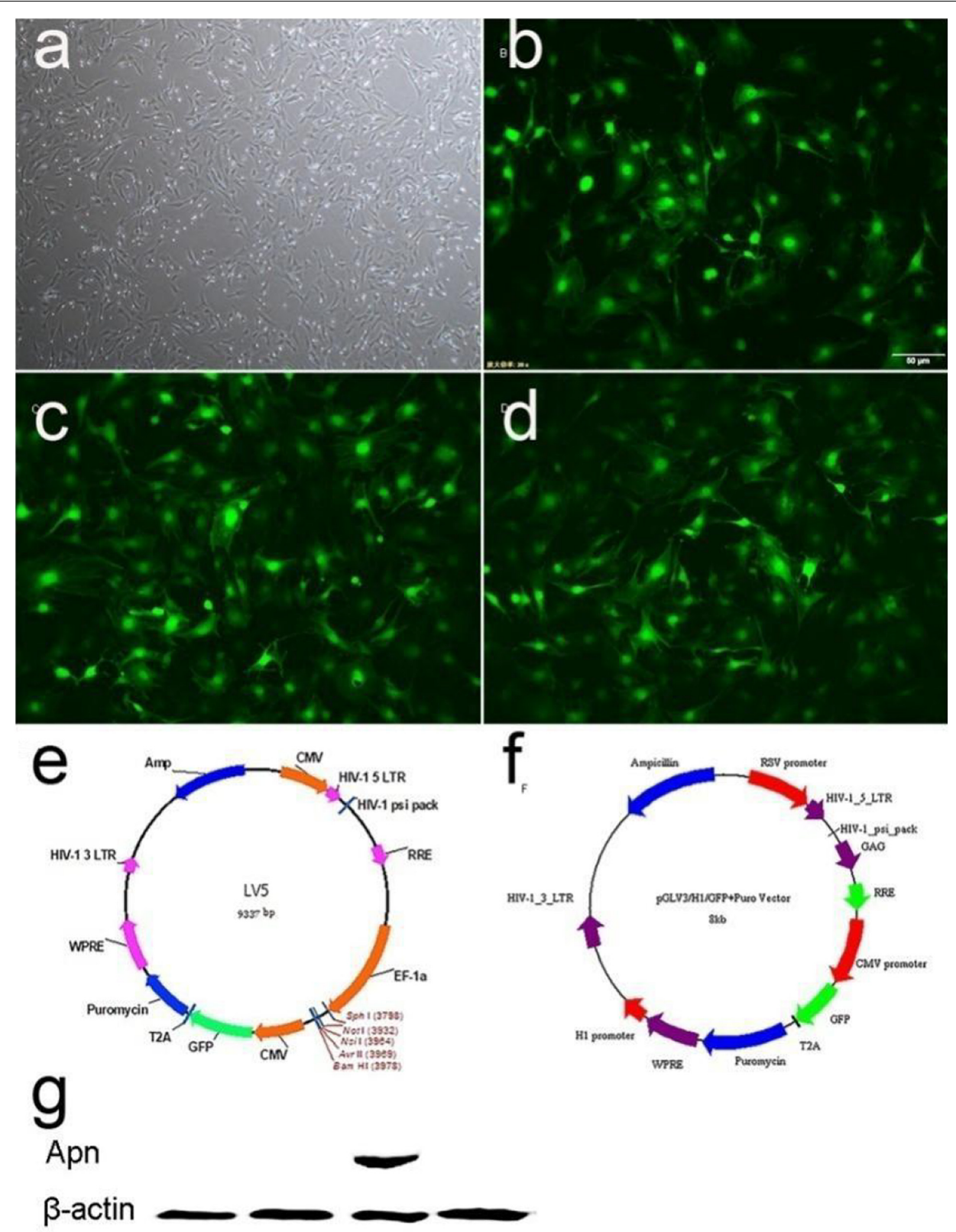

FIGURE 1 | The APN plasmid construction and expression. (a) Morphology characterization of cultured BMSCs (light microscopy). (b) GFP expression in BMSCs $48 \mathrm{~h}$ after transduction of empty vector into BMSCs. (c) GFP expression in BMSCs $48 \mathrm{~h}$ after transduction of APN into BMSCs. (d) GFP expression in BMSCs $48 \mathrm{~h}$ after transduction of APN-siRNA into BMSCs. (e) Maps of LV5. (f) Maps of LV3. (g) Western blotting detection of APN protein expression.

\section{RESULTS}

\section{The BMSCs Isolation and APN Modification}

The primary BMSCs were isolated and identified as our previous study (Wang et al., 2016). The GFP was used to detect the transfection efficiency of the virus (Figures 1e,f). A total of $48 \mathrm{~h}$ after infection, BMSCs were infected by lentivirus with GFP, and normal morphology was showed in three groups by fluorescence microscope (BX-53, Olympus
Corp., Tokyo, Japan) (Figures 1a-d). The results indicated over $80 \%$ transduction efficiency in BMSCs. A total of $72 \mathrm{~h}$ after transfection, the expression of APN was significantly increased in the BMSCs/APN+ group, while almost no detectable levels in the BMSCs, the BMSCs/Vehicle, and the BMSCs/APNgroup (Figure 1g).

\section{The Metabolic Indexes in All Groups}

Six weeks after treatment, the body weight of DM rats was lower than that of the control rats (Figure $2 \mathrm{~A}, p<0.01$ ), which 

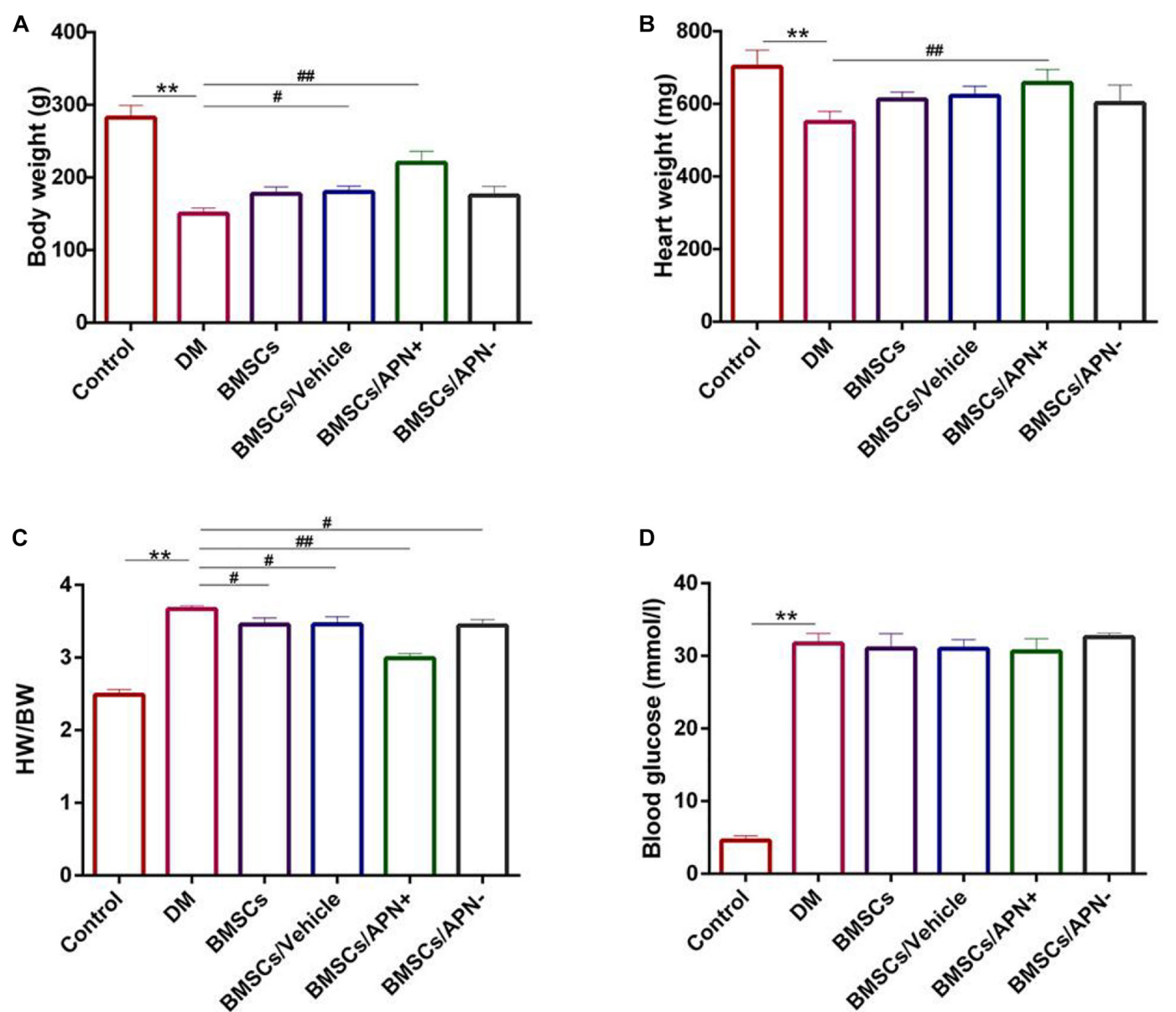

FIGURE 2 | Basic characteristics of experiment groups changes at 6 weeks. (A) Body weight (g). (B) Heart weight (mg). (C) Heart weight to body weight ratio (HW/BW). (D) Blood glucose (mmol/l). Date are mean $\pm \mathrm{SD}$; ${ }^{\star} p<0.05$, ${ }^{* *} p<0.01,{ }^{\#} P<0.05$, vs DM, ${ }^{\# \#} P<0.01$, vs DM.

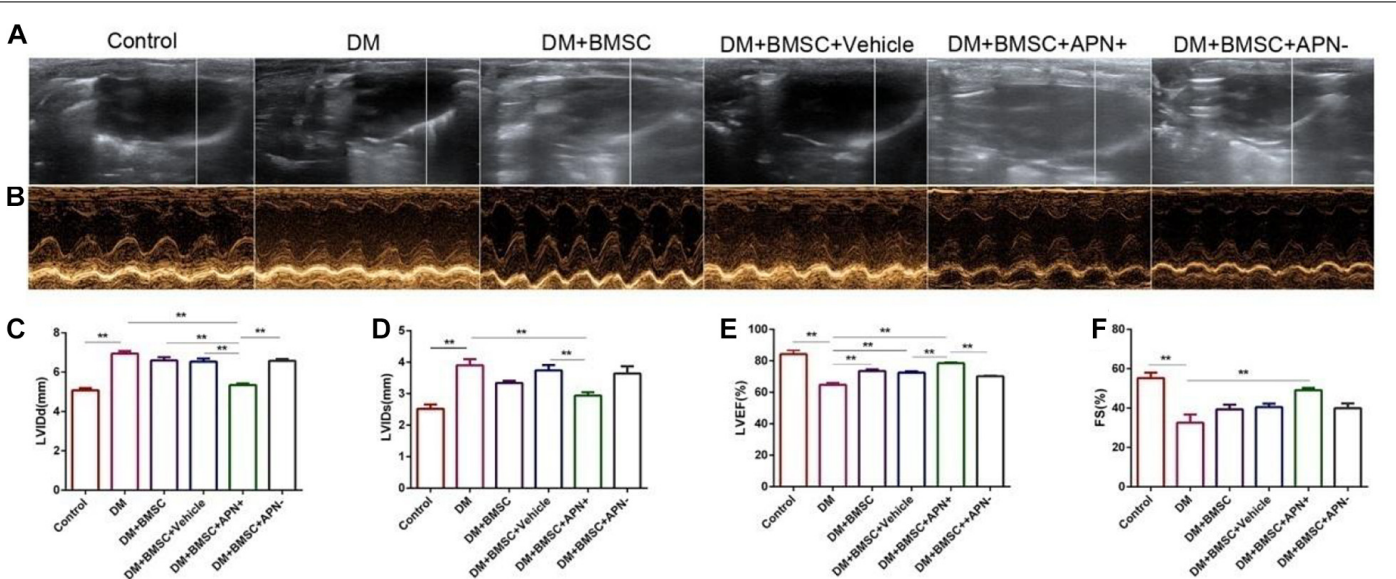

FIGURE 3 | Effects of APN on diabetes-induced LV dysfunction. (A) Two-dimensional echocardiograms. (B) M-mode echocardiograms. (C) Left ventricular internal diameter at end-diastole (LVIDD). (D) Left ventricular internal dimension systole (LVIDS). (E) LV ejection fraction (LVEF). (F) Fractional shortening (FS). Data are mean $\pm \mathrm{SD} ;{ }^{\star} p<0.05,{ }^{\star *} p<0.01$. 

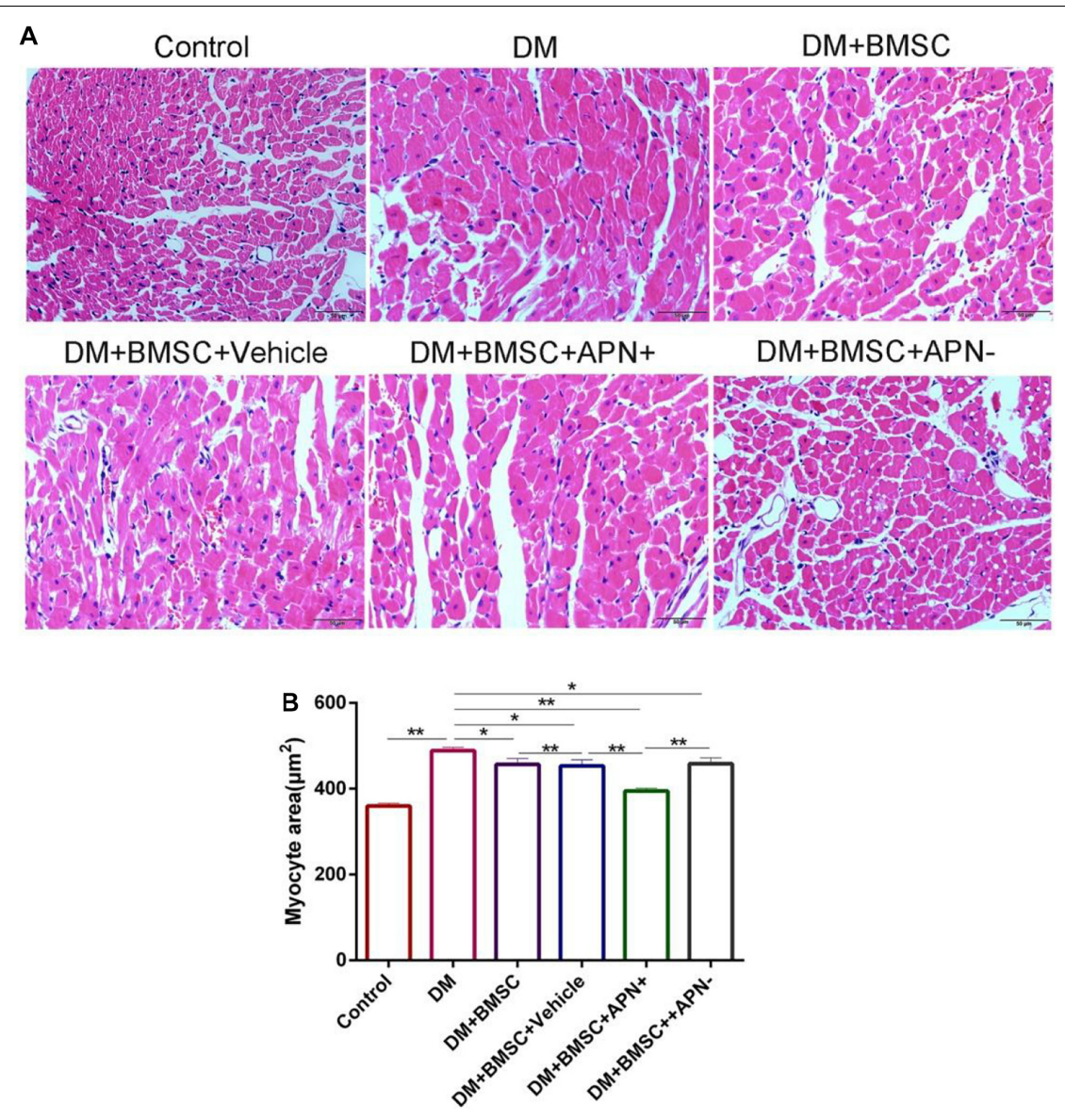

FIGURE 4 | APN attenuated pathological changes in the hearts of diabetic rats. (A) Representative micrographs of myocardial tissue sections stained with hematoxylin and eosin (scale bar: $50 \mu \mathrm{m})$. (B) Quantitative analysis of myocyte size area. Data are mean $\pm \mathrm{SD} ;{ }^{*} p<0.05,{ }^{* *} p<0.01$.

accompanied by the decrease of the heart weight (Figure 2B, $p<0.01)$. There is no remarkable differences in the BMSCs group, the BMSCs/Vehicle group and the BMSCs/APN- group. However, both of body weight and heart weight showed a significant increase in the BMSCs/APN+ group while comparing to the diabetic rats (Figures $2 \mathrm{~A}, \mathbf{B}, p<0.01$ ).

The ratio of heart weight to body weight $(\mathrm{mg} / \mathrm{g})$ was increased in the DM group and decreased in the BMSCs, the BMSCs/Vehicle, the BMSCs/APN- group, and BMSCs/APN+ group (Figure 2C, $p<0.01$ ). After STZ injection, the blood glucose increased while compared to the control rats. There are no significant differences in the rest groups (Figure 2D, $p>0.05$ ).

\section{APN Modified BMSCs Attenuated Diabetes-Induced Cardiac Dysfunction}

The cardiac structure and systolic/diastolic function in all rats were assessed by echocardiogram. Six weeks after treatment, the LVEF ratio was above 70 , and the FS ratio was more than 40 in the normal rats. The DM rats exhibited a larger LVIDD/s diameter $(p<0.01$, Figures 3A-D) and LVEF. However the FS ratio decreased $(p<0.01$, Figures $3 \mathbf{A}, \mathbf{E}, \mathbf{F})$ when compared to the control rats. BMSC/APN+ treatment dramatically improved diameter in LVIDD, LVIDs, LVEF, and FS ratio $(p<0.01$, Figure 3). There is no improvement of LVIDD and LVIDs in rats of BMSCs, BMSCs/Vehicle, and BMSCs/APN- group (Figure 3, $p>0.05$ ). Moreover, the LVEF ratio of the BMSCs group and BMSCs/Vehicle group also improved significantly (Figure 3, $p<0.01$ ) while the FS ratio of the BMSCs group, the BMSCs/Vehicle group, and the BMSCs/APN- group did not show significant alterations (Figures 3A,F, $p>0.05$ ).

\section{APN Modified BMSCs Improved Pathological Injury of Diabetic Heart}

We analyzed the pathological changes of myocardial tissue to investigate the benefit of BMSCs/APN+ on diabetesinduced myocardial structural injury. The results demonstrated that diabetic heart exhibited cardiomyocyte hypertrophy, irregular myocardial arrangements, increased interstitial spaces, and myofibrillar discontinuation (Figure 4A). The normal control heart had regular myocardial structures and clear visible nuclei. Furthermore, BMSCs/APN+ group rats could improve these pathological changes. Cross-sections of left ventricular myocardial were examined at a magnification of $400 \times$ (Figure 4B). The myocyte areas of LV were significantly 

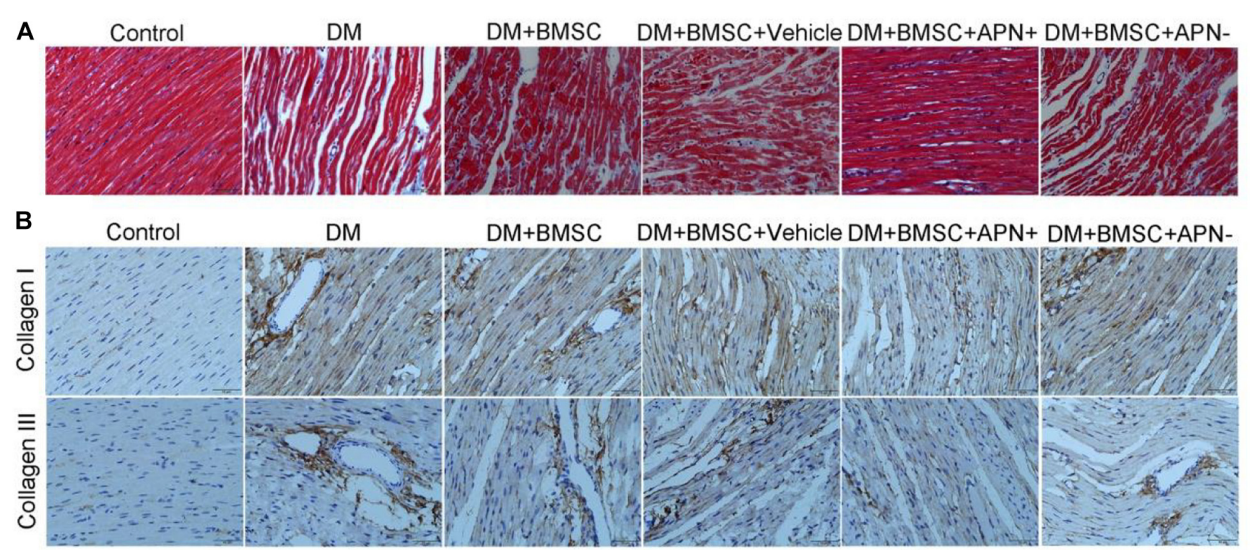

C
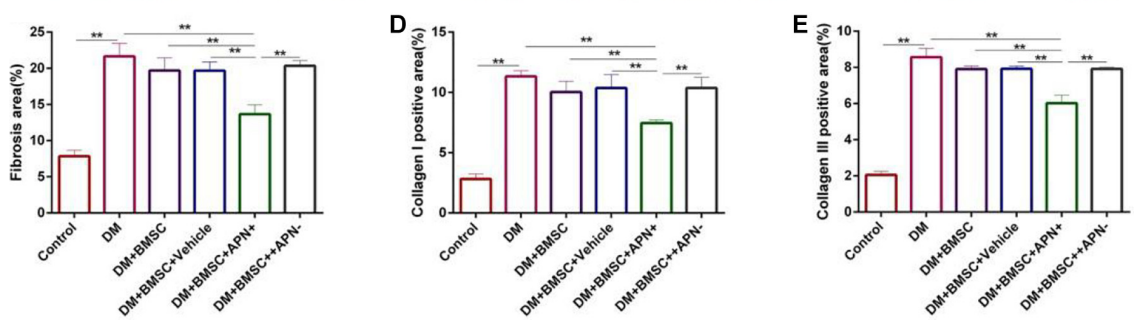

FIGURE 5 | Effect of APN on collagen deposition. (A) Representative Masson's trichrome staining. (B) Immunostaining of collagen I and collagen III. (C) Quantitative analysis of Masson's trichrome staining. (D) Immunohistochemistry analysis of collagens I. (E) Immunohistochemistry analysis of collagens III. Data are mean \pm SD; ${ }^{*} p<0.05,{ }^{* *} p<0.01$

increased in the DM rats, while compared with the control rats (Figure 4B, $p<0.01$ ). The results indicated that transplanted BMSCs could reduce the increasing myocyte size, especially in the BMSCs/APN+ treatment groups (Figure 4C, $p<0.01$ ).

\section{APN Modified BMSCs Improved Diabetes-Induced Myocardial Fibrosis}

Myocardial fibrosis is the major pathological lesion of DCM. Masson's trichrome staining of the heart showed certain irregularity of the myocardial fibrosis and increased collagen accumulation in the diabetes rats when compared with the normal heart. However, BMSCs/APN+ treated rats showed a remarked improvement for reduction collagen accumulation when compared to the diabetes rats (Figures 5A,C, $p<0.01$ ). The collagen I and III, as cardiac fibrotic markers, were augmented under diabetic conditions and BMSCs/APN+ could reduce collagens I and III expression (Figure 5, $p<0.01$ ). There is no significant differences of collagens I and III expression in the rest groups (Figure 5, $p>0.05$ ). These results demonstrated that BMSCs/APN+ could attenuate myocardial fibrosis and improve the collagen deposition in diabetic hearts.

\section{APN Modified BMSCs Alleviates Diabetes-Induced TGF- $\beta 1 / \mathrm{Smad} 2 / 3$ Signaling Pathway Activation}

To verify the possible signaling pathway involved in myocardial fibrosis of diabetic heat, we analyzed the expression of TGF$\beta / \mathrm{Smad} 2 / 3$ by immunohistochemistry. The results showed their expression in the nucleus (Figure 6A) and the TGF$\beta 1 / \mathrm{Smad} 2 / 3$ protein increased in diabetic hearts (Figure 6, $p<0.01$ ), while the BMSCs/APN+ treatment can reverse the these upregulation (Figure 6, $p<0.01$ ). However, there is no detectable difference in the rest three groups (Figure 6, $p>0.05)$. The western blot analysis demonstrated that the TGF- $\beta 1 / \mathrm{Smad} 2 / 3$ protein had significant reduction in the BMSCs/APN+ group (Figure 6, $p<0.01$ ), and no marked alteration in the BMSCs group, the BMSCs/Vehicle group, and the BMSCs/APN- group as an assumption (Figure 6, $p>0.05$ ). We also detected the expression of p-smad $2 / 3$ protein, and found it phosphorylation was positively stressed in the BMSCs/APN+ diabetic rats (Figure 6, $p<0.01$ ), but there is no significant improvement in the rest three groups (Figure 6, $p>0.05$ ). These results indicated that BMSCs/APN+ could inhibit cellular TGF- $\beta 1 / \mathrm{Smad} 2 / 3$ related signal pathways, which may alleviate diabetes-induced myocardial fibrosis in diabetic rats.

\section{BMSCs/APN+ Co-cultured With H9c2 Inhibited High Glucose Induced TGF- $\beta 1 /$ Smad2/3 Activation}

To clarify the role of BMSCs/APN+ treatment in collagen deposition in vitro, we cultured H9c2 in normal glucose (25 mM) as the control group and high glucose $(50 \mathrm{mM})$ as the DM group. H9c2 in high glucose co-cultured with the BMSCs, the BMSCs/Vehicle, the BMSCs/APN+, and the BMSCs/APNfor 7 days, respectively. The western blot analysis showed 


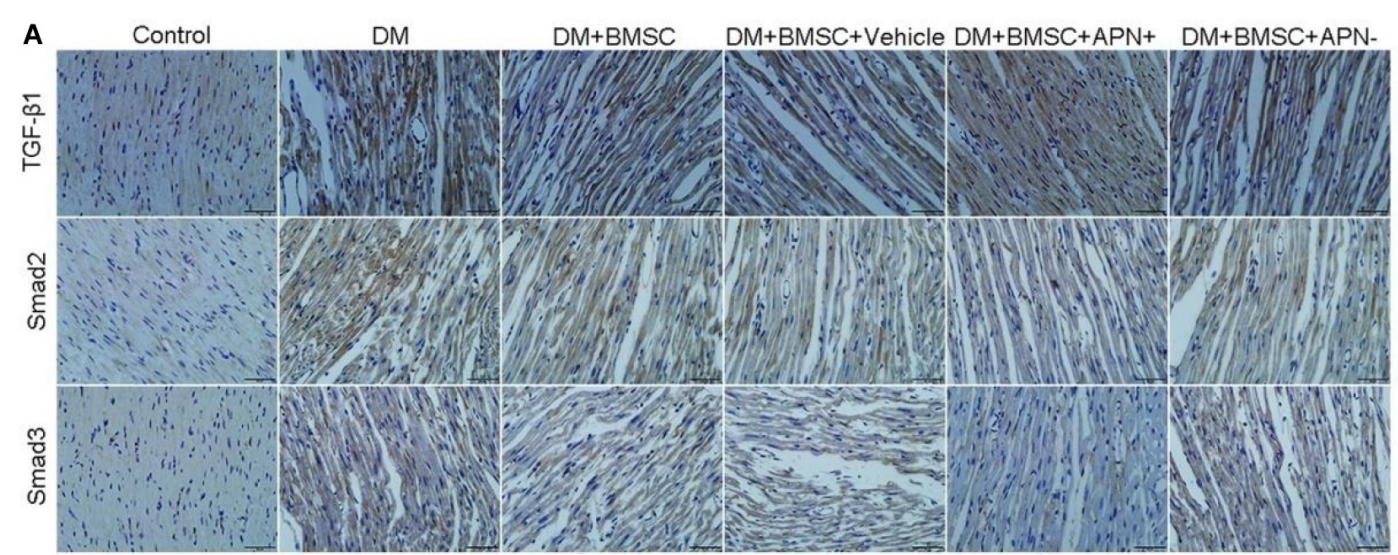

B

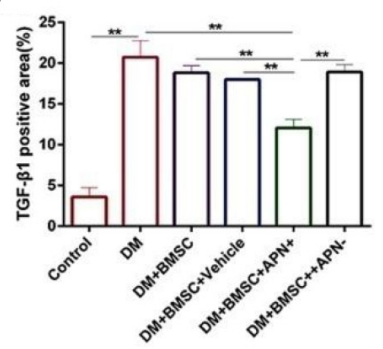

E

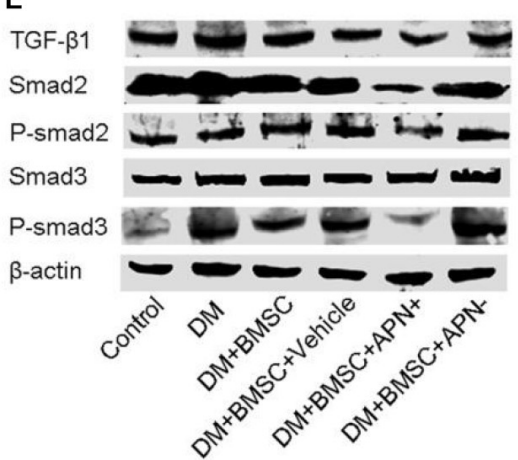

H

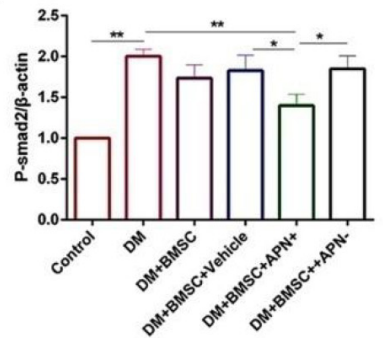

C

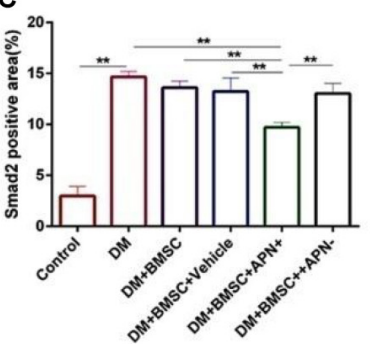

F
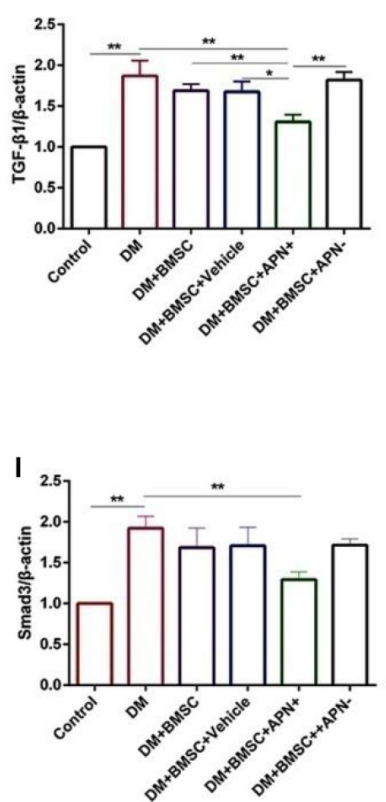

D

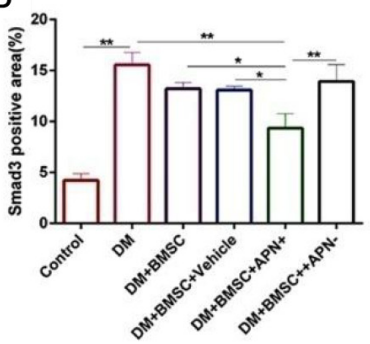

G
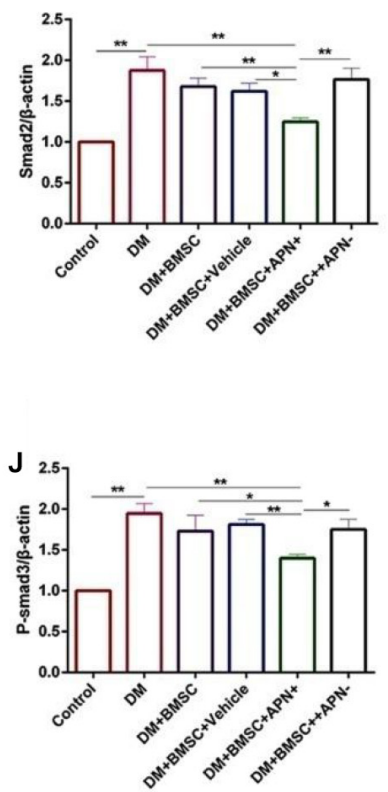

FIGURE 6 | The expression of TGF- $\beta 1 /$ Smad 2,3 in all groups rats. (A) Immunostaining of TGF- $\beta 1$, Smad2 and Smad3. (B) Quantitative analysis of TGF- $\beta 1$. (C) Quantitative analysis of Smad2. (D) Quantitative analysis of Smad3. (E) Representative western blot: TGF- $\beta 1$, Smad2, P-smad2, Smad3, and P-smad3. (F) The western blot assay of TGF- $\beta 1$. (G) The western blot assay of Smad2. (H) The western blot assay of P-smad2. (I) The western blot assay of Smad3. (J) The western blot assay of P-smad3. Data are mean $\pm \mathrm{SD} ;{ }^{*} p<0.05,{ }^{*} p<0.01$.

that $\mathrm{H} 9 \mathrm{c} 2$ cultured in high glucose significantly increased the TGF- $\beta 1$ Smad2, P-smad2, Smad3, and P-smad3 when compared with the normal control group (Figure 7, $p<0.01$ ). BMSCs/APN + could inhibit the expression of TGF- $\beta 1 / \mathrm{Smad} 2 / 3$ (Figure $7, p<0.01$ ). There were no significantly altered in the BMSCs, the BMSCs/Vehicle, and BMSCs/APN- treated $\mathrm{H} 9 \mathrm{c} 2$ cells while compared with the high glucose cultured cells (Figure 7, $p>0.05$ ). The present results demonstrated that BMSCs/APN+ could alleviate high glucose-induced TGF$\beta 1 / \mathrm{Smad} 2 / 3$ activation. 


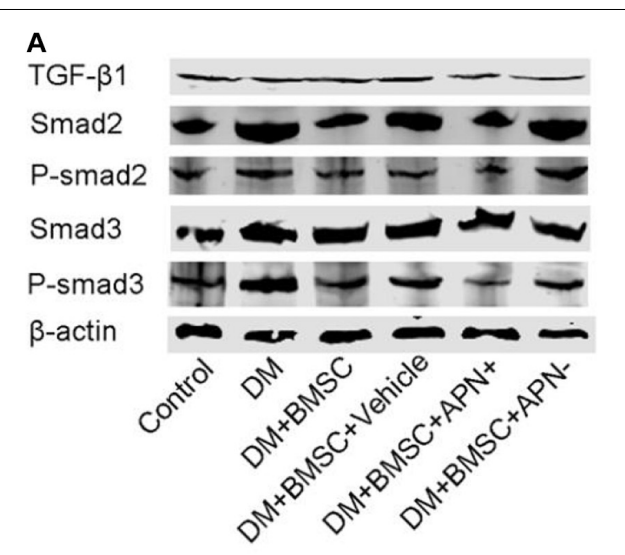

C

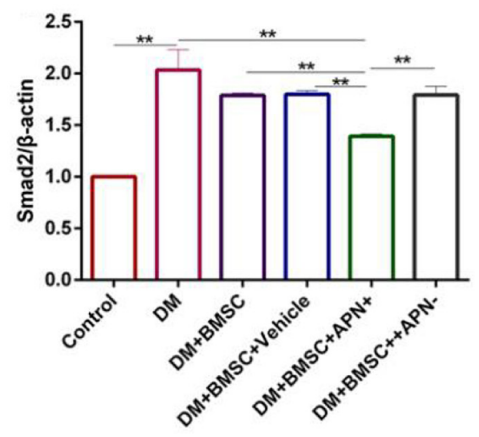

E

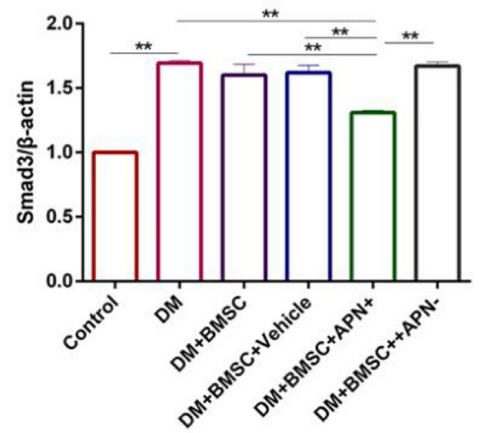

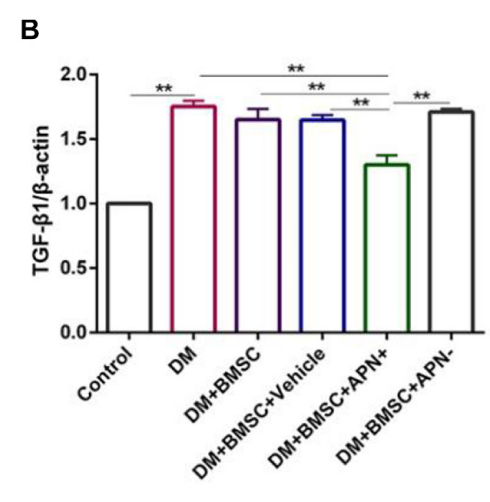

B
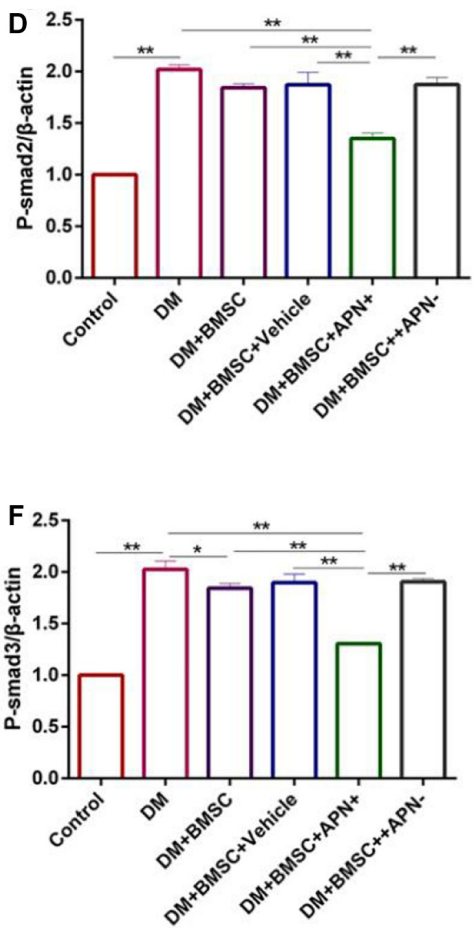

FIGURE 7 | The expression of TGF- $\beta 1$ /Smad 2,3 in high glucose cultured H9c2 cells. (A) Representative western blot: TGF- $\beta 1$, Smad2, p-smad2, Smad3, and p-smad3. (B) The western blot assay of TGF- $\beta 1$. (C) The western blot assay of Smad2. (D) The western blot assay of $p$-smad2. (E) The western blot assay of Smad3. (F) The western blot assay of $p$-smad3. Data are mean $\pm \mathrm{SD} ;{ }^{*} p<0.05,{ }^{* *} p<0.01$.

\section{DISCUSSION}

Cardiac fibrosis is one of the most common pathological lesions of DCM, ultimately leading to heart failure (Huynh et al., 2014). Therefore, reducing the excessive proliferation of collagen in the myocardium is necessary for preventing DCM and heart failure (Talior-Volodarsky et al., 2012). The present study for the first time suggested that APN modified BMSCs could improve fibrosis in diabetic heart in vitro and in vivo. During a 6-week followup period, we found a significant increase of collagen fibers and pathological changes in diabetic heart. The expression of collagen I and III also increased significantly in diabetic myocardium. The echocardiographic results showed that sustained increasing collagen fibers in the myocardium might damage the cardiac function. Interestingly, a tail-vein injection of BMSC/APN+ caused significant improvement of body weight, cardiac function, collagen accumulation, and attenuated the pathological lesions in diabetic hearts.

Myocardial fibrosis is a chronic and progressive process characterized by an excessive accumulation of collagen fibers in diabetic hearts (Luo et al., 2013). Cardiac fibrosis contributes to cardiac remodeling, and finally leads to the progression of heart failure. Under diabetes conditions, myocardial fibrosis is regulated by various factors. The previous study revealed that TGF- $\beta 1$ is the most important one (Sui et al., 2015). The studies of diabetic animals revealed that increased TGF- $\beta 1$ was 
associated with cardiac fibrosis. Moreover, TGF- $\beta$ inhibition can attenuate cardiac fibrosis in animal models (Avila et al., 2014). The scientists found that high glucose could increase TGF- $\beta 1$ activity and downstream canonical Smad signaling that increased interstitial fibrosis and cellular hypertrophy in diabetic hearts (Shen et al., 2014). Activated TGF- $\beta 1$ binds to the TGF- $\beta 1$ receptor, it will allow the phosphorylation of the Smad 2/3 proteins. These Smads can be directly activated through phosphorylation by the TGF- $\beta$ type I receptor kinase. Smad3 has been recognized as a key mediator of TGF$\beta$-induced pro-fibrotic outcomes. Both Smad2 and Smad3 have been implicated in ECM production and tissue fibrosis (Yuan et al., 2013). Subsequently, these Smads complexes can be translocated into the nucleus and then regulate the transcription of the target genes, such as pro-fibrotic genes, that play crucial roles in the development of myocardial fibrosis (Zhang et al., 2014).

In the present study, the immunohistochemistry and western blot assay results demonstrated that the expression of TGF$\beta 1, \operatorname{Smad} 2 / 3$, and $\mathrm{p}-\mathrm{Smad} 2 / 3$ protein in the diabetic hearts tended to increase. Furthermore, BMSCs/APN+ treatment could decrease Smad2/3 phosphorylation, be consistent with the improvement of collagen formation and cardiac function while BMSCs/APN - have no effects on cardiac fibrosis, functional recovery in the diabetic hearts. Under the condition of long-term high glucose stimulation, the expression of TGF- $\beta 1$ in cardiomyocytes increases, which may induce fibroblasts to synthesize a large amount of collagen, promote fibroblast proliferation, and transform into myofibroblasts (Zhang et al., 2014). The APN modified BMSCs may reduce myofibrillar disorder and inhibit the proliferation and transformation of cardiac fibroblasts, as well as the interaction between fibroblasts and cardiomyocytes via TGF- $\beta 1 /$ Smad signal pathway.

H9c2 cell line showed an undifferentiated phenotype, which was similar to the physiological characteristics of normal cardiomyocytes in morphology, biochemistry and other characteristics, and was easy to obtain, passable and stable, which makes up for the poor repeatability of primary cardiomyocytes. H9c2 cardiomyocytes also have some limitations when used in isolated heart models. The present data demonstrated that high glucose increased expression of TGF- $\beta 1$ and $\mathrm{p}-\mathrm{Smad} 2 / 3$ in $\mathrm{H} 9 \mathrm{c} 2$ cells while co-cultured with the BMSCs/APN+ could reduce the expression of TGF- $\beta 1$ and $\mathrm{p}-\mathrm{Smad} 2 / 3$. These results were consistent with the animal studies.

\section{REFERENCES}

American Diabetes Association (2012). Diagnosis and classification of diabetes mellitus. Diabetes Care 35(Suppl. 1), S64-S71.

Avila, G., Osornio-Garduno, D. S., Rios-Perez, E. B., and Ramos-Mondragon, R. (2014). Functional and structural impact of pirfenidone on the alterations of cardiac disease and diabetes mellitus. Cell Calcium 56, 428-435. doi: 10.1016/j. ceca.2014.07.008

Bugyei-Twum, A., Advani, A., Advani, S. L., Zhang, Y., Thai, K., Kelly, D. J., et al. (2014). High glucose induces Smad activation via the transcriptional coregulator p300 and contributes to cardiac fibrosis and hypertrophy. Cardiovasc. Diabetol. 13:89. doi: 10.1186/1475-2840-13-89

\section{CONCLUSION}

In summary, the present data demonstrate a protective role of BMSCs/APN+ on diabetic hearts. Interestingly, high glucose increased TGF- $\beta 1$, p-Smad $2 / 3$, and collagen fibers deposition while BMSCs/APN+ could suppress these trend and improve functional recovery in the hearts of diabetic rats. Therefore, the present study provided a novel strategy for the treatment of DCM in patients with diabetes.

\section{DATA AVAILABILITY STATEMENT}

The raw data supporting the conclusions of this article will be made available by the authors, without undue reservation.

\section{ETHICS STATEMENT}

The animal study was reviewed and approved by the Committee of Animal Experiment Center of Zhejiang University (Hangzhou, China).

\section{AUTHOR CONTRIBUTIONS}

KM performed the cells culture and animal model. KM and $\mathrm{HC}$ contributed to writing the manuscript and the data analysis. SC assisted with the experiments. $\mathrm{YH}$ and $\mathrm{XZ}$ conceived the study and contributed to the manuscript preparation. All authors read and approved the final version of the manuscript.

\section{FUNDING}

This work was supported by the Zhejiang Provincial Natural Science Foundation of China (Grant No. LY18H170002) and the National Natural Science Foundation of China (Grant No. 81472149).

\section{ACKNOWLEDGMENTS}

The authors thank Meilin $\mathrm{Tu}$ for the Echocardiography assay. The authors thank all the participants for their support in this study.

Cavender, M. A., Steg, P. G., Smith, S. C. Jr., Eagle, K., Ohman, E. M., Goto, S., et al. (2015). Impact of diabetes mellitus on hospitalization for heart failure, cardiovascular events, and death: outcomes at 4 years from the reduction of atherothrombosis for continued health (REACH) registry. Circulation 132, 923-931. doi: 10.1161/circulationaha.114. 014796

Chang, W. T., Cheng, J. T., and Chen, Z. C. (2016). Telmisartan improves cardiac fibrosis in diabetes through peroxisome proliferator activated receptor delta (PPARdelta): from bedside to bench. Cardiovasc. Diabetol. 15:113.

Falcao-Pires, I., Hamdani, N., Borbely, A., Gavina, C., Schalkwijk, C. G., van der Velden, J., et al. (2011). Diabetes mellitus worsens diastolic left ventricular dysfunction in aortic stenosis through altered myocardial structure 
and cardiomyocyte stiffness. Circulation 124, 1151-1159. doi: 10.1161/ circulationaha.111.025270

Faramoushi, M., Amir Sasan, R., Sari Sarraf, V., and Karimi, P. (2016). Cardiac fibrosis and down regulation of GLUT4 in experimental diabetic cardiomyopathy are ameliorated by chronic exposures to intermittent altitude. J. Cardiovasc. Thorac. Res. 8, 26-33. doi: 10.15171/jcvtr. 2016.05

Hasegawa, Y., Kishimoto, S., Shibatani, N., Nomura, H., Ishii, Y., Onishi, M., et al. (2010). The pharmacokinetics of morphine and its glucuronide conjugate in a rat model of streptozotocin-induced diabetes and the expression of MRP2, MRP3 and UGT2B1 in the liver. J. Pharm. Pharmacol. 62, 310-314. doi: 10.1211/jpp.62.03.0004

Huynh, K., Bernardo, B. C., McMullen, J. R., and Ritchie, R. H. (2014). Diabetic cardiomyopathy: mechanisms and new treatment strategies targeting antioxidant signaling pathways. Pharmacol. Ther. 142, 375-415. doi: 10.1016/j. pharmthera.2014.01.003

Jia, G., Hill, M. A., and Sowers, J. R. (2018a). Diabetic cardiomyopathy: an update of mechanisms contributing to this clinical entity. Circ. Res. 122, 624-638. doi: 10.1161/circresaha.117.311586

Jia, G., Whaley-Connell, A., and Sowers, J. R. (2018b). Diabetic cardiomyopathy: a hyperglycaemia- and insulin-resistance-induced heart disease. Diabetologia 61, 21-28. doi: 10.1007/s00125-017-4390-4

Jing, Z., Qiong, Z., Yonggang, W., and Yanping, L. (2014). Rat bone marrow mesenchymal stem cells improve regeneration of thin endometrium in rat. Fertil. Steril. 101, 587-594. doi: 10.1016/j.fertnstert.2013.10.053

Koitabashi, N., Danner, T., Zaiman, A. L., Pinto, Y. M., Rowell, J., Mankowski, J., et al. (2011). Pivotal role of cardiomyocyte TGF-beta signaling in the murine pathological response to sustained pressure overload. J. Clin. Invest. 121, 2301-2312. doi: 10.1172/jci44824

Li, J. H., Zhang, N., and Wang, J. A. (2008). Improved anti-apoptotic and antiremodeling potency of bone marrow mesenchymal stem cells by anoxic preconditioning in diabetic cardiomyopathy. J. Endocrinol. Invest. 31, 103-110. doi: 10.1007/bf03345575

Luo, M., Guan, X., Luczak, E. D., Lang, D., Kutschke, W., Gao, Z., et al. (2013). Diabetes increases mortality after myocardial infarction by oxidizing CaMKII. J. Clin. Invest. 123, 1262-1274. doi: 10.1172/jci65268

Nishihara, T., Matsuda, M., Araki, H., Oshima, K., Kihara, S., Funahashi, T., et al. (2006). Effect of adiponectin on murine colitis induced by dextran sulfate sodium. Gastroenterology 131, 853-861. doi: 10.1053/j.gastro.2006.06.015

Oruqaj, G., Karnati, S., Vijayan, V., Kotarkonda, L. K., Boateng, E., Zhang, W., et al. (2015). Compromised peroxisomes in idiopathic pulmonary fibrosis, a vicious cycle inducing a higher fibrotic response via TGF-beta signaling. Proc. Natl. Acad. Sci. U.S.A. 112, E2048-E2057.

Sanber, K. S., Knight, S. B., Stephen, S. L., Bailey, R., Escors, D., Minshull, J., et al. (2015). Construction of stable packaging cell lines for clinical lentiviral vector production. Sci. Rep. 5:9021.

Scherrer-Crosbie, M., Steudel, W., Hunziker, P. R., Foster, G. P., Garrido, L., Liel-Cohen, N., et al. (1998). Determination of right ventricular structure and function in normoxic and hypoxic mice: a transesophageal echocardiographic study. Circulation 98, 1015-1021. doi: 10.1161/01.cir.98.10.1015

Seferovic, P. M., and Paulus, W. J. (2015). Clinical diabetic cardiomyopathy: a two-faced disease with restrictive and dilated phenotypes. Eur. Heart J. 36, 1718-1727. doi: 10.1093/eurheartj/ehv134
Shen, N., Li, X., Zhou, T., Bilal, M. U., Du, N., Hu, Y., et al. (2014). Shensong Yangxin Capsule prevents diabetic myocardial fibrosis by inhibiting TGFbeta1/Smad signaling. J. Ethnopharmacol. 157, 161-170. doi: 10.1016/j.jep. 2014.09.035

Sui, X., Wei, H., and Wang, D. (2015). Novel mechanism of cardiac protection by valsartan. synergetic roles of TGF-beta1 and HIF-1alpha in Ang II-mediated fibrosis after myocardial infarction. J. Cell. Mol. Med. 19, 1773-1782. doi: $10.1111 /$ jcmm. 12551

Talior-Volodarsky, I., Connelly, K. A., Arora, P. D., Gullberg, D., and McCulloch, C. A. (2012). alpha11 integrin stimulates myofibroblast differentiation in diabetic cardiomyopathy. Cardiovasc. Res. 96, 265-275. doi: 10.1093/cvr/ cvs259

Tian, M., Tang, L., Wu, Y., Beddhu, S., and Huang, Y. (2018). Adiponectin attenuates kidney injury and fibrosis in deoxycorticosterone acetate-salt and angiotensin II-induced CKD mice. Am. J. Physiol. Renal. Physiol. 315, F558F571.

Travis, M. A., and Sheppard, D. (2014). TGF-beta activation and function in immunity. Annu. Rev. Immunol. 32, 51-82. doi: 10.1146/annurev-immunol032713-120257

Wang, C., Shi, D., Song, X., Chen, Y., Wang, L., and Zhang, X. (2016). Calpain inhibitor attenuates ER stress-induced apoptosis in injured spinal cord after bone mesenchymal stem cells transplantation. Neurochem. Int. 97, 15-25. doi: 10.1016/j.neuint.2016.04.015

Wang, T., Mao, X., Li, H., Qiao, S., Xu, A., Wang, J., et al. (2013). NAcetylcysteine and allopurinol up-regulated the Jak/STAT3 and PI3K/Akt pathways via adiponectin and attenuated myocardial postischemic injury in diabetes. Free Radic. Biol. Med. 63, 291-303. doi: 10.1016/j.freeradbiomed.2013. 05.043

Wang, W. K., Wang, B., Lu, Q. H., Zhang, W., Qin, W. D., Liu, X. J., et al. (2014). Inhibition of high-mobility group box 1 improves myocardial fibrosis and dysfunction in diabetic cardiomyopathy. Int. J. Cardiol. 172, 202-212. doi: 10.1016/j.ijcard.2014.01.011

Wang, Y., Yang, H., Nolan, M., Burgess, J., Negishi, K., and Marwick, T. H. (2018). Association of depression with evolution of heart failure in patients with type 2 diabetes mellitus. Cardiovasc. Diabetol. 17:19.

Yuan, H. F., Huang, H., Li, X. Y., Guo, W., Xing, W., Sun, Z. Y., et al. (2013). A dual AP-1 and SMAD decoy ODN suppresses tissue fibrosis and scarring in mice. J. Invest. Dermatol. 133, 1080-1087. doi: 10.1038/jid.2012.443

Zhang, Y., Shao, L., Ma, A., Guan, G., Wang, J., Wang, Y., et al. (2014). Telmisartan delays myocardial fibrosis in rats with hypertensive left ventricular hypertrophy by TGF-beta1/Smad signal pathway. Hypertens. Res. 37, 43-49. doi: 10.1038/ hr.2013.119

Conflict of Interest: The authors declare that the research was conducted in the absence of any commercial or financial relationships that could be construed as a potential conflict of interest.

Copyright (C) 2021 Meng, Cai, Cai, Hong and Zhang. This is an open-access article distributed under the terms of the Creative Commons Attribution License (CC BY). The use, distribution or reproduction in other forums is permitted, provided the original author(s) and the copyright owner(s) are credited and that the original publication in this journal is cited, in accordance with accepted academic practice. No use, distribution or reproduction is permitted which does not comply with these terms. 